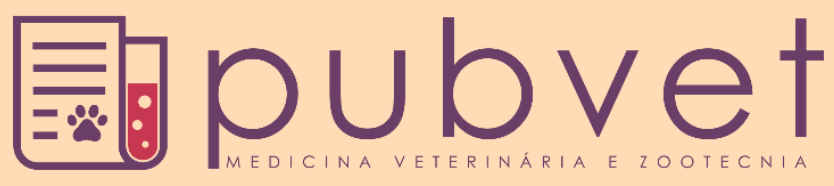

https://doi.org/10.31533/pubvet.v15n10a930.1-7

\title{
Diagnósticos e tratamentos empregados em casos de hérnias de disco em cães: Revisão
}

\author{
Aline Rodrigues Guidi ${ }^{1}$, Júlia Paçô Castedo ${ }^{1 *}$, Lilian Câmara De Paula Santos ${ }^{1 \bullet}$, Alysson \\ Rodrigo Lamounier ${ }^{2} \theta$ \\ ${ }^{1}$ Discentes do curso de Medicina Veterinária na Pontifícia Universidade Católica de Minas Gerais, Instituto de Ciências \\ Biológicas e da Saúde, Unidade Praça da Liberdade. \\ ${ }^{2}$ Docente do curso de Medicina Veterinária na Pontifícia Universidade Católica de Minas Gerais, Instituto de Ciências \\ Biológicas e da Saúde, Unidades Praça da Liberdade e Betim. \\ *Autor para correspondência, E-mail: juliapcastedo@gmail.com
}

\begin{abstract}
Resumo. Para a execução do trabalho, foram realizadas pesquisas em artigos científicos a fim de apresentar dados atuais sobre as cirurgias de hérnias de disco, distúrbio esse o maior causador de alterações neurológicas em cães. Primeiramente classificada por Hansen, a doença do disco intervertebral (DDIV) apresenta vários motivos, seja por extrusão compressiva ou não do núcleo pulposo, por protusão do disco ou por trauma. Pode ser diagnosticada após anamnese, exames físicos e de imagem e tratada apenas por medicamentos, cirurgicamente e, em seu pós-operatório, por fisioterapia. É uma alteração bastante comum em cães, em especial nas raças condrodistróficas, sendo de suma importância na ortopedia veterinária.
\end{abstract}

Palavras-chave: Animais domésticos, discopatias, terapêutica

\section{Diagnoses and treatments used in cases of herniated discs in dogs: Review}

\begin{abstract}
For the execution of this study, research was carried out on scientific articles in order to present current data on herniated disc surgery, a disorder that is the biggest cause of neurological disorders in dogs. Firstly, classified by Hansen, intervertebral disc disease (DDIV) has several reasons, whether by compressive extrusion of the pulpal nucleus, by protrusion of the disc or by trauma. It can be diagnosed after anamnesis, physical and imaging exams, and treated with medication, surgically and, in post operation, by physiotherapy. It is a very common alteration in dogs, especially in chondrodystrophic breeds, being of extreme importance in veterinary orthopedics.
\end{abstract}

Key words: Discopathies, domestic animals, therapeutic

\section{Introdução}

A hérnia de disco é a causa mais comum de injúria à medula espinhal (Alves, 2018) e de alterações neurológicas em cães (Silva, 2017). Esta alteração ocorre devido a degeneração ou lesão das camadas do disco intervertebral (núcleo pulposo e anel fibroso), tanto em raças condrodistróficas, quanto em raças não condrodistróficas. As raças condrodistróficas, tais como Dachshund, Beagle e Poodle, podem apresentar alterações no núcleo pulposo desde os três meses de idade (Cecim, 2019), com isso apresentam maior chance de ocorrer exteriorização do mesmo após uma ruptura do anel fibroso. Ao contrário, as raças não condrodistróficas costumam mostrar essa afecção em idade mais avançada (Coelho et al., 2016; Jones et al., 2000; McGavin \& Zachay, 2013), podendo sofrer apenas protusão do disco (Rodrigues, 2016). 
Há vários motivos pelo qual as hérnias discais se manifestam. Pode ser por degeneração ou metaplasia condróide (Hansen tipo 1), ou por degeneração fibrosa ou metaplasia fibróide (Hansen tipo 2). Além disso, podem mostrar-se após lesões traumáticas (herniação do tipo 3), o que é comum em idosos de raças distróficas (Nerone \& Diamante, 2018). É possível também, por fim, se apresentar como um material anular ou nuclear no canal vertebral (hérnia tipo sequestrada) (Vilanova Junior et al., 2019). Após detectada essa patologia, seja por radiografia, tomografia computadorizada, mielografia ou ressonância magnética, é de suma importância que os tratamentos sejam iniciados. Dentre eles, o medicamentoso, o cirúrgico e o fisioterápico são os indicados, sendo o último mais adequado no póscirúrgico.

\section{Anatomia da coluna vertebral}

A coluna vertebral é composta por vértebras, que são diferenciadas de acordo com a região a qual estão localizadas. Por exemplo, a vértebra da região cervical, é diferente da vértebra da região lombar. Apesar disso, todas elas são compostas pelo corpo, a parte ventral cilíndrica, sobre a qual se sustentam as demais partes, com parte cranial convexa e parte caudal côncava cobertas por cartilagem hialina; o arco vertebral, se formando sobre a face dorsal do corpo, delimitando o forame vertebral; e os processos vertebrais, utilizados para a fixação de músculos e ligamentos. Ainda, os forames vertebrais de cada vértebra são responsáveis por circundar a medula espinhal, suas meninges, nervos espinhais, vasos sanguíneos, ligamentos, tecido adiposo e tecido conectivo, portanto, consegue proteger essas estruturas de traumas e lesões (Köning \& Liebich, 2011; Reece, 2008; Sisson et al., 1986).

Dessa forma, duas vértebras imediatas são intercaladas por um disco, denominado de disco intervertebral, sendo esse composto por um núcleo pulposo e um anel fibroso, que, por sua vez, é recoberto por tecido fibroso (Figura 1).

A disposição anatômica das fibras, em formas espirais ao redor do eixo longitudinal das vértebras, permite maior estabilidade do disco, atuando juntamente com os ligamentos longitudinais dorsais e ventrais (Figura 2), visando evitar danos e alterações nessas estruturas, como a hérnia de disco (Köning \& Liebich, 2011; Reece, 2008; Sisson et al., 1986).

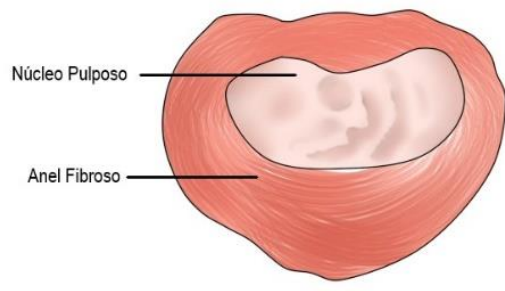

Figura 1. Disco intervertebral da região lombar de um cão. Fonte: Adaptado por Júlia Paçô a partir de Hans-Georg (2016).

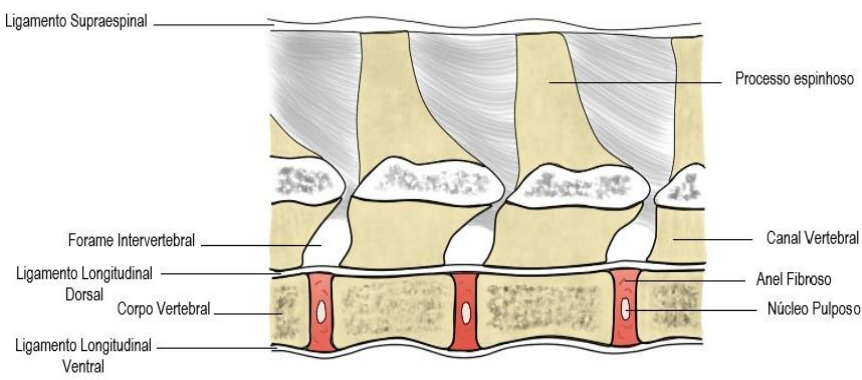

Figura 2. Ligamentos longos e curtos da coluna lombar (representação esquemática, secção paramediana) segundo Ghetie, 1954. Fonte: Adaptado por Júlia Paçô a partir de Hans-Georg (2016)

\section{Hérnia de disco intervertebral}

De forma geral, as hérnias de disco são associadas à degeneração dos discos intervertebrais, causando a exteriorização do núcleo pulposo para o canal vertebral (Alves, 2018; Thrall, 2013). É uma doença que acomete principalmente cães condrodistróficos, devido à predisposição de metaplasia óssea, que pode atingir o anel cartilaginoso, provocando a ruptura deste. Cães não condrodistróficos podem sofrer apenas a protusão do disco, ou seja, sem ocorrer a ruptura do anel, ocorrência que não é considerada como a verdadeira hérnia de disco (Coelho et al., 2016; Ferreira Neto et al., 1977; McGavin \& Zachay, 2013). Na região cervical, os ligamentos são mais amplos espessos que no restante da coluna, por isso as extrusões laterais de discos são mais comuns nessa região (Alves, 2018; Thrall, 2013).

A doença do disco intervertebral (DDIV) foi primeiramente classificada por Hansen, por isso, suas diferentes classificações levam seu nome. A DDIV Hansen tipo 1 (Figura 3A) ocorre por processo degenerativo, causando alteração na concentração de glicosaminoglicanos, perda de água e de proteoglicanos e o aumento do teor de colágeno. Com isso, o disco intervertebral passa a ser mais 
cartilaginoso e seu núcleo mais granular, e a consequente tendência à mineralização faz com que o material perca a capacidade de absorver impacto. Nesse tipo de DDIV, ocorre a extrusão do material, sendo um processo crônico, é observado em animais jovens de até cinco anos. A herniação do tipo 2 (Figura 3B) é caracterizada pela protrusão do disco causada por desvio de material do núcleo, estando associada à degeneração fibroide. É mais observada em animais não condrodistróficos, ocorrendo entre $8 \mathrm{e}$ 10 anos de idade (Alves, 2018; Thrall, 2013).

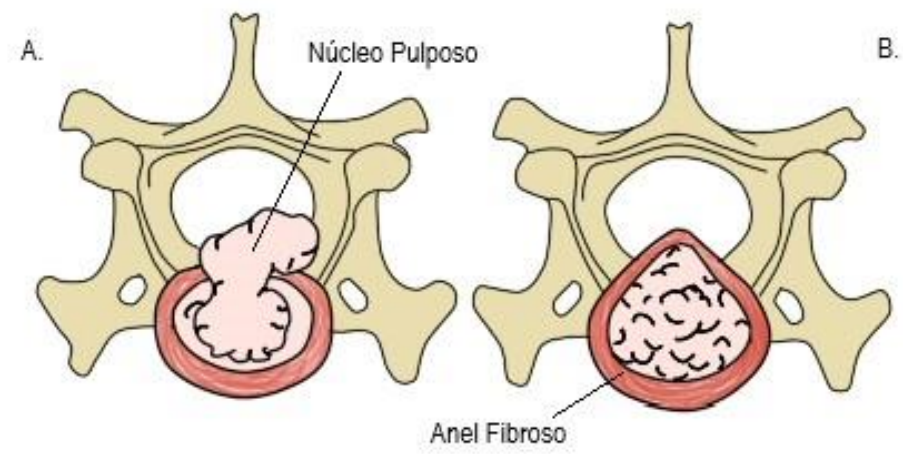

Figura 3. Demonstração gráfica discopatia Hansen tipo 1 (A) e discopatia Hansen tipo 2 (B). Fonte: Adaptado por Júlia Paçô a partir de Kistemacher (2017).

A DDIV tipo 3 nunca foi descrita por Hansen, mas é didaticamente descrita como discopatia de Hansen tipo 3. Tais hérnias são associadas à traumas e exercícios vigorosos, provocando a ruptura do ânulo fibroso dorsal e consequente extrusão do núcleo pulposo (Alves, 2018; Thrall, 2013). Nesse caso, a extrusão do disco atinge a medula em alta velocidade, com isso causa contusão no parênquima medular e se dissipa pelo espaço epidural, sem causar compressão. Pode acometer animais de qualquer idade, porém, é comumente observada em animais mais velhos de raças condrodistróficas. Esses animais podem apresentar dor à plegia, de acordo com o grau e local da lesão (Nerone \& Diamante, 2018).

A hérnia de disco sequestrada (Figura 4), por sua vez, é caracterizada pela presença de material no ânulo fibroso que é mais confinado ao disco (Levine \& Fingeroth, 2015; Vilanova Junior et al., 2019). E o nódulo de Schmorl (Figura 5) é definido como herniação focal do disco por meio da placa terminal, adentrando o corpo vertebral. Em sua maioria, esses nódulos são assintomáticos, sendo achados acidentalmente em exames de imagem. Em alguns casos, pode causar dor lombar e afetar o sistema nociceptivo (Kim \& Jang, 2018). Ambos os tipos de hérnias supracitados são raros de ocorrer, tanto em cães quanto em gatos (Vilanova Junior et al., 2019).

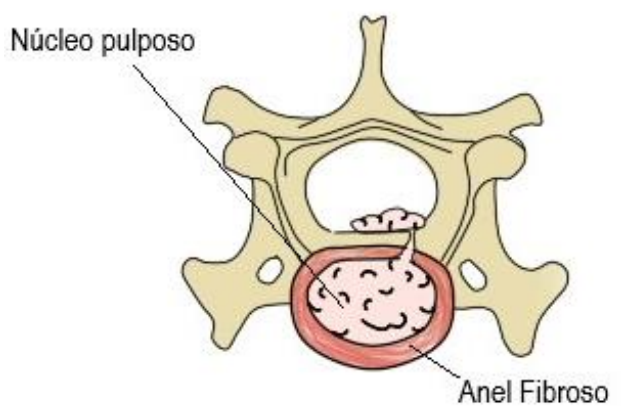

Figura 4. Demonstração gráfica hérnia de disco sequestrada. Fonte: Adaptado por Júlia Paçô a partir de Baurfeind Brasil; e Kistemacher (2017).

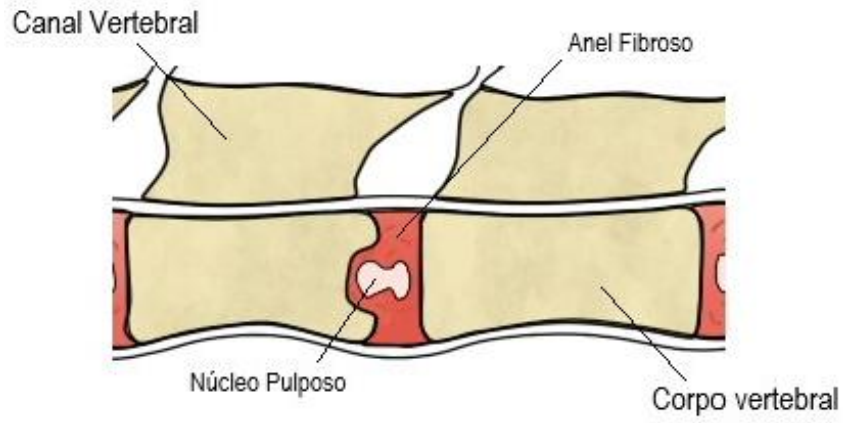

Figura 5. Demonstração gráfica Nódulo de Schmorl. Fonte: Adaptado por Júlia Paçô a partir de Hans-Georg e Kim \& Jang (2018)

\section{Diagnóstico da DDIV}

O diagnóstico da DDIV é baseado na anamnese e histórico do paciente, considerando principalmente predisposição de acordo com a espécie e raça do animal; assim como exame neurológico e exames complementares (Silveira et al., 2020). No exame neurológico, avalia-se a integridade dos componentes, levando em consideração que se que detectar um distúrbio neurológico, qual sua localização e extensão. 
Doenças inflamatórias podem ser consideradas ao montar um plano de diagnósticos diferenciais, como meningite ou meningoencefalite granulomatosa, por isso são pedidos exames hematológicos e de líquor (Cesca, 2018). Para um diagnóstico conclusivo, exames de imagem como radiografia, tomografia computadorizada, mielografia, ou ressonância magnética são essenciais (Alves, 2018; Cesca, 2018). Dentre esses, o exame mais indicado é o de ressonância magnética, tendo em vista que o corte transversal permite localizar e quantificar a compressão medular (Alves, 2018) e, além de fornecer detalhes anatômicos superiores a outros exames (Cesca, 2018), permite também verificar se há apenas um ou mais discos herniados.

É importante ressaltar que, dos exames de imagem citados anteriormente, no Brasil o de melhor acesso, tanto por disponibilidade do aparelho quanto por custo do exame, é a radiografia. Isso se dá pelo pequeno número de cidades que dispõem dos aparelhos de ressonância magnética e de tomografia computadorizada. Ademais, a mielografia apresenta riscos aos pacientes, por utilizar anestesia geral e contrastes à base de iodo, e não permite fechar o diagnóstico concreto, tendo em vista que casos de edema da medula não permitem identificação correta do local afetado (Cesca, 2018). Por isso, nem sempre é possível ter um diagnóstico completo nos casos de DDIV, uma vez que na radiografia é possível apenas ver alterações no exame por diminuição do espaço intervertebral, o que não necessariamente indica ocorrência da hérnia (Alves, 2018; Cesca, 2018).

\section{Tratamento cirúrgico}

O tratamento cirúrgico é indicado em casos nos quais o animal não responde bem ao tratamento medicamentoso, assim como quando a compressão medular compromete de forma significativa a qualidade de vida do animal, em casos de grande quantidade de material extravasado (Moschen, 2017), com isso, o principal objetivo das cirurgias é remover o material degenerado, permitindo, assim, descompressão do canal. Existem vários tipos de cirurgias que podem ser feitas em casos de hérnias de disco, sendo necessária a avaliação do médico veterinário responsável para definir qual a melhor opção para o quadro apresentado. Entre elas, em casos de hérnias de disco cervicais podem ser feitas a fenda ventral, laminectomia dorsal, hemilaminectomia, mini-hemilaminectomia ou pediculectomia parcial (Silveira et al., 2020), e entre as técnicas utilizadas para tratamento de hérnia de disco toracolombar, a mais comum é a corpectomia parcial.

A fenda ventral (ou slot ventral) permite visualização ventral da região acometida, por isso não é indicada para lesões lateralizadas (Schwab et al., 2020). Essa técnica é realizada por meio da fenestração dos discos acometidos pela ressecção do anel ventral, de forma a expor o núcleo pulposo (Padilha Filho \& Selmi, 1999). A fenestração consiste na remoção de uma região do anel fibroso em formato de retângulo (Gusmão, 2021). Então, são removidas as camadas ósseas, e é feita a incisão do ligamento longitudinal dorsal, permitindo, assim, visualização do canal vertebral e remoção do material extrusado (Padilha Filho \& Selmi, 1999).

A cirurgia de laminectomia dorsal envolve a retirada dos processos espinhosos dorsais, lâminas dorsais e algumas partes dos processos articulares e pedículos de, no mínimo, duas vértebras consecutivas. Permite acesso aos dois lados do canal vertebral, porém não oferece vantagens a mais, quando comparada à outras técnicas (Moschen, 2017). Nessa técnica, há uma grande chance de ser feita a remoção incompleta do material herniado, por isso não é aconselhada pela maioria dos autores (Gusmão, 2021).

A técnica de hemilaminectomia é baseada na excisão em apenas um dos lados dos processos articulares, tanto cranial quanto caudal, assim como da lâmina óssea e pedículos das vértebras envolvidas. A principal vantagem desse método é a manutenção da estrutura e mecânica vertebral, tendo em vista que é menos traumática que outras técnicas (Silveira et al., 2020). Além disso, essa técnica permite melhor acesso em hérnias mais dorsais, e boa visualização da medula espinhal e nervos. Como desvantagens, pode ocorrer deterioração dos sinais neurológicos (Veiga, 2018). Essa técnica é indicada em casos de compressão medular por lesão no canal vertebral lateral, dorsolateral ou ventrolateral, podendo ser feita bilateralmente (Santos, 2017).

Por outro lado, técnica de mini-hemilaminectomia (ou pediculotomia parcial) é feita com duas incisões cutâneas, sendo cada uma delas dois espaços vertebrais cranial e caudal ao local afetado. Com 
a vértebra isolada, o processo acessório é removido com auxílio de uma broca. Então, é possível remover o material herniado (Gusmão, 2021). A região ventral que passará por cirurgia é limitada pela zona ventral do forâmen (Gusmão, 2021), por isso, a principal desvantagem dessa técnica é o risco de hemorragia junto à estrutura, assim como permitir acesso limitado ao canal vertebral. Apesar disso, em hérnias com uma posição mais ventral, permite melhor acesso, com risco mínimo de trauma iatrogênico e menor trauma nos tecidos adjacentes. Em comparação à hemilaminectomia, requer menos tempo de cirurgia, portanto o animal passa menos tempo sob efeitos de anestesia geral, e a recuperação póscirúrgica é mais rápida (Veiga, 2018).

Na medicina veterinária, a técnica de corpectomia parcial é mais utilizada em casos crônicos de hérnias de disco toracolombares (Gonzalez, 2017). O acesso cirúrgico pode ser tanto dorsolateral quanto ventrolateral. Essa técnica envolve a remoção parcial dos corpos vertebrais que suportam a zona herniada, evitando, assim, a manipulação da medula espinhal (Gusmão, 2021); por meio de um "slot" perfurado no corpo da vértebra com base na extensão da compressão provocada. As principal vantagem dessa cirurgia é a baixa reincidência de hérnias no local operado, uma vez que, quando feita de forma mecânica, a fenestração permite remoção mais completa do núcleo (Marinho et al., 2018).

\section{Tratamento fisioterápico}

O tratamento fisioterápico é utilizado principalmente no pós-operatório, sendo iniciado o mais cedo possível. É feito com o objetivo de fortalecer a musculatura dos membros, e encorajar seu uso (Moschen, 2017). A partir disso, a fisioterapia é indicada em casos de hérnias de disco, pós-operatório de cirurgias ortopédicas, traumas agudos, apresentando também benefícios como redução da dor e do tempo de recuperação, além de melhora na qualidade de vida (Alves, 2018; Kistemacher, 2017).

Nos casos de hérnias discais do tipo 1, logo após a cirurgia o animal deve ficar em repouso absoluto de 2 a 4 semanas, o que provoca atrofia da musculatura. Com isso, o tratamento fisioterápico, nos primeiros 10 dias de pós-operatório visam a manutenção das funções urinária e fecal, e da cicatrização tecidual. Após o $10^{\circ}$ dia, espera-se remissão dos sintomas de dor e inflamação, sendo adicionados protocolos da atividade física, como a esteira aquática, de forma a estimular a coordenação e reação de postura; e deslocamento de pesos, de forma a fortalecer a musculatura (Silva, 2017).

Normalmente, em casos de discopatias a amplitude dos membros é afetada, por isso são indicados alongamentos associados com exercícios de amplitude, de forma a melhorar a flexibilidade das articulações e extensibilidade dos tecidos periarticulares e tendões. As massagens, muito utilizadas em tratamentos fisioterápicos, auxiliam na recirculação de líquidos intersticiais para os vasos linfáticos, e possivelmente ajude na circulação sanguínea, melhorando também o transporte de nutrientes. Com isso, previne formação de tecido fibrosos, e remove produtos das reações inflamatórias, auxiliando no controle da dor crônica (Festugatto et al., 2008; Ramalho et al., 2015).

Ainda, pode-se utilizar de eletroestimulação, que pode ser aplicada para provocar contrações musculares, ou, em casos cervicais, utilizar da modalidade TENS (em inglês, Transcutaneal Electrical Nerve Stimulation), que promove analgesia e pode ser utilizado diariamente. O laser terapêutico é aplicado de forma focal, tendo ação analgésica e anti-inflamatória, permitindo, assim redução do uso de medicamentos; e a termoterapia permite aumento da velocidade de condução de impulsos nervosos, devido à ação vasodilatadora (Festugatto et al., 2008; Kistemacher, 2017; Ramalho et al., 2015). A cinesioterapia, área de maior estudo na reabilitação veterinária, é aplicada de acordo com a evolução do quadro clínico do paciente, auxiliando na melhora da força, mobilidade, flexibilidade e coordenação (Ramalho et al., 2015). A hidroterapia, por fim, além de melhorar a amplitude dos movimentos, auxilia também no aumento de força muscular e de massa, aliviando o uso de força direta sobre os ossos e articulações. É contraindicada em casos de dor aguda, e em casos de hérnias cervicais deve ser utilizada com cautela, em momentos mais avançados do tratamento (Alves, 2018; Festugatto et al., 2008; Kistemacher, 2017; Ramalho et al., 2015; Silva, 2017).

\section{Considerações finais}

A doença do disco intervertebral é causa frequente de lesão medular em cães e acomete principalmente cães condrodistróficos. Cães sem esse perfil podem sofrer apenas a protusão do disco 
que o não caracteriza verdadeira hérnia de disco. Quando da anamnese, o veterinário além de colher informações sobre o histórico do paciente deve considerar a predisposição em relação à espécie e raça do animal e solicitar exame neurológico, hematológico e de líquor para descartar diagnósticos diferenciais, como meningite ou meningoencefalite granulomatosa. Destaca-se que para um diagnóstico conclusivo, exames de imagem são essenciais e, o mais indicado é o de ressonância magnética, porque o corte transversal permite localizar e quantificar a compressão medular, além de verificar se há apenas um ou mais discos herniados. Considerando o tratamento cirúrgico, diversas técnicas podem ser adotadas para a remoção o material degenerado, descompressão do canal, sendo necessária a avaliação do médico veterinário responsável para definir a melhor opção para o paciente. Qualquer que seja a modalidade cirúrgica empregada, o animal deve sempre beneficiar de um protocolo de reabilitação podendo ser indicado tratamento fisioterápico, alongamentos associados com exercícios de amplitude, massagens, eletroestimulação, laser terapêutico, cinesioterapia e hidroterapia.

\section{Referências bibliográficas}

Alves, L. S. (2018). Diagnóstico por imagem de hérnia discal hansen tipo i, ii e iii em cães. Veterinaria e Zootecnia, 25(1), 10-21. https://doi.org/10.35172/rvz.2018.v25.35.

Cecim, B. F. (2019). Doença do disco intervertebral em cães da raça Dachshund: Uma revisão de literatura. Iniciação Científica Cesumar, 21(2), 189-201. https://doi.org/10.17765/15181243.2019v21n2p189-201.

Cesca, P. H. (2018). Doença do disco intervertebral cervical em cães. Universidade Federal do Rio Grande do Sul.

Coelho, H. E., Santos, R. L., \& Alessi, A. C. (2016). Patologia veterinária. Coelho.

Ferreira Neto, J. M., Viana, J. M. M. E. S., \& Magalhães, L. M. (1977). Patologia clínica veterinária. Rabelo e Brasil.

Festugatto, R., Mazzanti, A., Raiser, A. G., Pelizzari, C., Salbego, F. Z., Beckmann, D. V., Pereira, D. T., \& Santos, R. P. (2008). Recuperação funcional de cães com doença do disco intervertebral toracolombar submetidos ao tratamento cirúrgico. Ciência Rural, 38(8), 2232-2238.

Gonzalez, P. C. S. Corpectomia vertebral cervical como uma técnica de descompressão medular em cães: estudo em cadáveres após o descongelamento. Universidade Federal do Rio Grande do Sul, Faculdade de Veterinária, Programa de Pós-Graduação em Ciências Vterinárias. Porto Alegre, 2017.

Gusmão, R. R. V. A. (2021). Resolução de hérnias discais por mínima invasão. Universidade de Lisboa, Faculdade de Medicina Veterinária.

Hans-Georg, K.H. L. Anatomia dos Animais Domésticos. Grupo Artmed. $6^{a}$ edição, c. 1, p. 90-91. 2016.

Hans-Georg, K.H. L. Anatomia dos Animais Domésticos. Grupo Artmed. 6a edição, c.1, p. 111 - 113. 2016.

Jones, T. C., D., H. R., \& King, N. W. (2000). Patologia veterinária. Manole.

Kim, S., \& Jang, S. (2018). Radicular pain caused by Schmorl's node: a case report. Revista Brasileira de Anestesiologia, 68(3), 322-324. https://doi.org/10.1016/j.bjan.2017.07.007.

Kistemacher, B. G. (2017). Tratamento fisioterápico na reabilitação de cães com afecções em coluna vertebral: revisão de literatura. Universidade Federal do Rio Grande do Sul.

Köning, H. E., \& Liebich, H. G. (2011). Anatomia dos animais domésticos texto e atlas colorido. Editora Artmed.

Levine, J. M., \& Fingeroth, J. M. (2015). Historical and current nomenclature associated with intervertebral disc pathology. Advances in Intervertebral Disc Disease in Dogs and Cats, 25-30. https://doi.org/10.1002/9781118940372.ch4.

Marinho, P. V. T., Ferrigno, C. R. A., Bregadioli, T., Paes, F., Santos, J. F., Macedo, A. S., Dal-Bó, Í. S., \& Galeazzi, V. S. (2018). Corpectomia lateral parcial para o tratamento de compressão ventral extradural crônica entre T9-T10 em um cão. Arquivo Brasileiro de Medicina Veterinária e Zootecnia, 70, 443-449.

McGavin, D., \& Zachay, J. F. (2013). Bases da patologia em veterinária. Elsevier Brasil.

Moschen, L. (2017). Doença do disco intervertebral cervical e toracolombar em pequenos animais. Universidade Federal do Rio Grande do Sul. 
Nerone, M. C., \& Diamante, G. A. C. (2018). Hérnia de disco tipo III em um cão. Acta Scientiae Veterinariae, 46(1), 350.

Padilha Filho, J. G., \& Selmi, A. L. (1999). Discopatia cervical no cão: tratamento cirúrgico através de fenestração ventral. Estudo retrospectivo (1986-1997). Ciência Rural, 29, 75-78.

Ramalho, F. P., Formenton, M. R., Isola, J. G. M. P., \& Joaquim, J. F. G. (2015). Tratamento de doença de disco intervertebral em cão com fisioterapia e reabilitação veterinária: relato de caso. Revista de Educação Continuada Em Medicina Veterinária e Zootecnia Do CRMV-SP, 13(1), 10-17.

Reece, W. O. (2008). Anatomia funcional e fisiologia dos animais domésticos. Editora Roca.

Rodrigues, M. I. V. (2016). Hérnia discal intervertebral tipo II toracolombar em cães: diagnóstico e tratamento cirúrgico de sete casos clínicos. Universidade de Lisboa.

Santos, J. F. (2017). Estudo biomecânico ex vivo de hemilaminectomia e pediculectomia em coluna toracolombar canina. Tese de Doutorado. Universidade de São Paulo, Faculdade de Medicina Veterinária e Zootecnia, Programa de Pós-Graduação, Departamento de Cirurgia.

Schwab, M. L., Ferrarin, D. A., Ripplinger, A., Herculano, L. F. S., Colvero, A. C. T., Wrzesinski, M. R., Rauber, J. S., Moro, S. S., Aiello, G., \& Mazzanti, A. (2020). Ventral slot in the clinical recovery of dogs with lateralized compression as a result of cervical intervertebral disc disease-20 cases (20082018). Ciência Rural, 50(8), 1-7. https://doi.org/10.1590/0103-8478cr20190242.

Silva, V. F. N. (2017). Fisioterapia como tratamento pós-cirúrgico de cães com hérnia de disco Hansen tipo I. Universidade Federal do Rio Grande do Sul.

Silveira, S. D., Batschke, C. F., Minuzzo, T., Correa, F. L., \& Rorig, M. C. L. (2020). Hemilaminectomia como tratamento de discopatia toracolombar canina: Estudo retrospectivo e relato de caso. PUBVET, 14(4), 1-12. https://doi.org/10.31533/pubvet.v14n4a544.1-123.

Sisson, S., Grossman, J. D., \& Getty, R. (1986). Anatomia dos animais domésticos. Interamericana.

Thrall, D. E. (2013). Textbook of veterinary diagnostic radiology. Elsevier Health Sciences.

Veiga, J. G. (2018). Comparação da evolução e tempo de recuperação entre hemilaminectomia e minihemilaminectomiano tratamento de hérnias discais torácicas elou lombares no cão. Coimbra.

Vilanova Junior, J. A., Dipp, G., Silva Neto, B. P., Balardini, J. L. M., Isaka, L. J. E., Jair, M. S. O. R., \& Engracia Filho, R. (2019). Hérnia de disco sequestrada em cão. Acta Scientiae Veterinariae, 47(1), 456.

Histórico do artigo:

Recebido: 15 de abril de 2021

Aprovado: 9 de junho de 2021
Licenciamento: Este artigo é publicado na modalidade Acesso Aberto sob a licença Creative Commons Atribuição 4.0 (CC-BY 4.0), a qual permite uso irrestrito, distribuição, reprodução em qualquer meio, desde que o autor e a fonte sejam devidamente creditados. 